\title{
Investigation of Some Coagulation Parameters in Pregnant Womens with Preeclampsia
}

\author{
Awad-Elkareem Abass ${ }^{1,2}$, Elsadig Adam ${ }^{1}$, Haitham Badwi ${ }^{1}$, Ali Hassan ${ }^{1}$, \\ Reem Mohamed ${ }^{1}$, Eiman Izzaldein ${ }^{1}$, Ayat Awad ${ }^{1}$. \\ ${ }^{1}$ Hematology department, Faculty of Medical Laboratory Sciences/ University of Khartoum, Sudan \\ ${ }^{2}$ Faculty of Medicine and Applied Medical Sciences/Northern Border University, KSA
}

\begin{abstract}
Background/Aim: Pre-eclampsia (PE) is a major obstetric health problem in developing countries and considers as one of the main causes of maternal mortality. The study aimed to investigate changes in some coagulation parameters in PE patients compared with normal pregnancies.

Methods: A prospective case control study was conducted in Omdurman Maternity Hospital-Khartoum state; in a period of March to June 2016. Pregnant women's with 28 weeks' gestation or more who admitted into the Department of Obstetrics and Gynaecology or attended at antenatal care unit, were enrolled in the study. A total of 60 pregnant women's; among them 30 patients with either mild or sever PE and 30 normal pregnancies as controls. Apparently healthy non-pregnant women's (N/30) also were included. Citrated venous blood samples were collected from each subject; platelet poor plasma was separated for measurement of prothombin time (PT), activated partial thromboplastin time (APTT), and Fibrinogen level by automated Stago analyser.

Results: In the present study the mean Fibrinogen level in the three studied groups was 3.96 $\pm 0.81,3.80 \pm 0.40$,

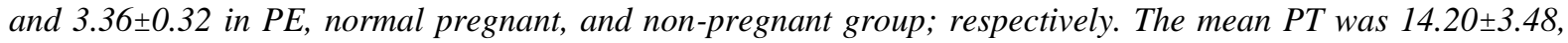
$12.90 \pm 1.13$, and $11.73 \pm 1.55$, whereas mean APTT was 38.32 $\pm 7.71,35.60 \pm 6.96$, and 33.56 \pm 6.26 ; respectively. The present study found that PT $(P ; 0.01,0.06)$, APPT $(P ; 0.02,0.04)$ of PE group was significantly higher than normal pregnant and non-pregnant groups; respectively. Fibrinogen of PE also was significantly higher than non-pregnant group $(P ; 0.003)$, however there was no significant variation with normal pregnant group $(P$; 0.3). Fifty percent of PE patients showed prolonged APTT, , 36.6\% prolonged PT, and $10 \%$ hypofibrinogenemia. However, Prolonged PT, APTT, and hypofibrinogenemia was seen in 3 cases of severe PE patients.
\end{abstract}

Conclusion: At late stage of pregnancy, the APTT, PT prolonged in PE pregnancy compared to normal pregnancy. However fibrinogen decreased in some cases of severe PE.

Keywords: Coagulation, Eclampsia, Fibrinogen, Hypertension, Pre-eclampsia, Pregnancy.

\section{I.Introduction}

Pre-eclampsia (PE) is a major obstetric disorder affecting between (5-8 \%) of pregnancies worldwide [1], [2]. Generally the diagnosis depends mainly on finding of hypertension and proteinuria after 20 weeks of pregnancy [3]. It considered as one of the main health problems associated with pregnancy and has high maternal mortality rate $[4,5]$. The prevalence of PE in developing countries ranges from $1.8 \%$ to $16.7 \%$ [6]. In Sudan there is high incidence of maternal mortality with PE, and accounting $4.2 \%$ of all obstetric complications and $18.1 \%$ of maternal deaths [7]. Although the exact cause of PE is not fully clarified, certain factors have been attributed to it which includes changes in placental perfusion, with changes in the coagulation system, endothelial dysfunction, fibrin deposition, and platelet activation $[6,8,9,10]$. PE can be classified as mild or severe PE, each one has different clinical manifestations and treatments [11]. It is essential to predict the severity of PE for rational gestational management [12].

Normal pregnancy is associated with some haemostatic changes; with features of increasing in several clotting proteins including fibrinogen, decreasing in the concentration of natural anticoagulants, and less fibrinolytic activity [13-15]. These changes result in a hypercoagulable state [13, 15], are likely due to hormonal changes [16] and increase the risk of thrombosis. This hypercoagulability is more at the time of delivery with release of thromboplastin substance due to placental expulsion [13]. Thromboplastin initiates clot formation to arrest maternal blood loss and reduce postpartum haemorrhage [17]. Generally, these haemostatic changes return to pre-pregnant levels within approximately one month postpartum [13, 14].

There is a definite exaggeration of the hypercoagulability during PE pregnancy. In PE, the coagulationfibrinolytic system is thought to be one of the most seriously affected systems by maternal inflammation [16]. The coagulation-anticoagulation balance is important for the regulation of utero-placental circulation and organ perfusion during pregnancy. When this balance is down-regulated in PE, the bloodstream of the placenta and 
many organs is blocked by microthrombi [18]. The super-hypercoagulable state of PE may also lead to disorder of metabolism as well as multiple organ dysfunction and may even complicate maternal and fetal lives. Therefore, coagulation testing is common in these patients for evidence of DIC [19,20] and HELLP (hemolysis, enzyme elevation and low platelet) syndrome). Thus, The study aimed to investigate changes in some coagulation parameters in a group of Sudanese pregnant women with PE and compared them with normal pregnancies.

\section{II.Material and Methods}

A case control study was conducted in Omdurman Maternity Hospital, Omdurman during the period of March 2016 to June 2016. Women with pregnancy of 28 weeks or more who attended at antenatal care unit or admitted into the Department of Obstetrics and Gynaecology, were enrolled in the study. Participants (N/60) were divided into two groups; PE group as patients(N/30), and normal pregnancy group (30) as controls. Apparently healthy non-pregnant women's (N/30) also were included. The Demographic and clinical data of each patient and control were recorded in a pre-designed a questionnaire.

Inclusion criteria: Mild or sever PE were diagnosed according to the standard diagnostic criteria; mild PE was defined as new onset of blood pressure $\geq 140 / 90 \mathrm{mmHg}$ after 20 weeks gestation, combined with proteinuria $\geq 0.3 \mathrm{~g} / 24 \mathrm{~h}$. Severe PE was defined as blood pressure $\geq 160 / 110 \mathrm{mmHg}$, serious proteinuria $(\geq 2 \mathrm{~g} / 24 \mathrm{~h})$ and manifestations of multiple organ damage or dysfunction. Patients with chronic renal/liver disease, diabetes mellitus, known bleeding disorder, and history of thrombosis or anticoagulant drug/oral contraceptive-use, were excluded from the study. Normal pregnancies were all healthy women had a normal obstetric history, with normal blood pressure, and had no proteinuria.

The research protocol was approved by the department of Haematology Review Board at Faculty of Medical Laboratory Sciences, Khartoum University. Informed written consent was taken from all participants. General physical and clinical examination was done at Hospital to obtain information about height, weight, systolic and diastolic blood pressure, oedema, pulse, and else. A 24 hour urine protein was performed as part of diagnostic work-up for PE.

Methods: From each enrolled subject citrated venous blood sample was collected, platelet poor plasma was separated immediately for coagulation analysis of PT, APTT, and fibrinogen level by semi-automated coagulometer (Stago analyser).

Statistical Analysis: The results data was collected and analysed using Statistical software 'Statistical Package for Social Science (SPSS) program for windows version 16 and Microsoft office Excell (2007). All quantitative variables were expressed as mean $\pm(\mathrm{SD})$ for each group. Simple T-test was used to test these quantitative parameters between the studied groups. Statistical significance was considered at $\mathrm{p}<0.05$.

\section{III.Results}

Demographic and clinical data of the participants are presented in Table 1. The difference in age and gestational period among the studied groups (PE and normal pregnancy) showed no statistical significance (Table 1. P; 0.3, 0.4, respectively). However, there is significant differences in body mass index (BMI) between two groups (Table 1. P; 0.02). As expected PE group patients had significantly higher systolic and diastolic blood pressure than healthy pregnant women (Table 1. P; 0.00001, 0.00001).

There were significant differences between the PT (P; 0.01, 0.06) and APTT (P; 0.02, 0.04) of PE group and normal pregnant/non-pregnant group, respectively (Table 2.). Fibrinogen of PE also was significantly differed with non-pregnant group (P; 0.003), however there was no significant variation with normal pregnant group (P; 0.3).

The mean Fibrinogen levels in the three studied groups was $3.96 \pm 0.81,3.80 \pm 0.40$, and $3.36 \pm 0.32$ in PE, normal pregnant, and non-pregnant group; respectively. The mean PT was $14.20 \pm 3.48,12.90 \pm 1.13$, and 11.73 \pm 1.55 , whereas mean APTT was $38.32 \pm 7.71,35.60 \pm 6.96$, and $33.56 \pm 6.26$; respectively. Fifty percent of PE patients showed prolonged APTT, , 36.6\% prolonged PT, and 10\% hypofibrinogenemia. Prolonged PT, APTT, and hypofibrinogenemia was seen in 3 cases of severe PE patients.

Table 1.Means \pm SD of Demographic/Clinical Data of Studied Pregnant Groups.

\begin{tabular}{|l|l|l|l|}
\hline Variable & PE Pregnancy (N/30) & Normal Pregnancy (N/30) & P. value \\
\hline Age/year & $28.18 \pm 6.44$ & $26.15 \pm 6.10$ & 0.30 \\
\hline Gestation period/week & $32.13 \pm 4.19$ & $33.42 \pm 2.85$ & 0.40 \\
\hline Systolic B.P/mmHg & $159.12 \pm 22.15$ & $105.18 \pm 13.14$ & $<.00001^{*}$ \\
\hline Diastolic B.P/ mmHg & $107.41 \pm 11.55$ & $72.62 \pm 10.30$ & $<.00001^{*}$ \\
\hline BMI (Kg/M2) & $28.10 \pm 4.20$ & $25.25 \pm 3.41$ & $0.02^{*}$ \\
\hline
\end{tabular}


Table 2. Means \pm SD of PT, APTT And Fibrinogen of the Three Studied Groups.

\begin{tabular}{|l|l|l|l|l|}
\hline Variable & PE Pregnant(N/30) & Normal Pregnant (N/30) & Non-Pregnant(N/30) & $\begin{array}{l}\text { P. } \\
\text { value }\end{array}$ \\
\hline PT & $14.20 \pm 3.48$ & $12.90 \pm 1.13$ & $11.73 \pm 1.55$ & $0.006^{*}$ \\
& & & & $0.001^{*}$ \\
\hline APTT & $38.32 \pm 7.71$ & $35.60 \pm 6.96$ & $33.56 \pm 6.26$ & $0.04^{*}$ \\
& & & & $0.02^{*}$ \\
\hline Fibrinogen & $3.96 \pm 0.81$ & $3.80 \pm 0.40$ & $3.36 \pm 0.32$ & $0.003^{*}$ \\
& & & 0.30 \\
\hline
\end{tabular}

\section{IV.Discussion}

PE and eclampsia remains one of the causes of perinatal mortality and maternal death in most developing countries. Many theories suggested that abnormal placentation is one of the critical issue in the pathogenesis of PE [suggested that platelet activation begins at initial duration of pregnancy in women with risk for PE [21].

This study compared some coagulation parameters of PT, APTT, and fibrinogen among two groups of pregnant women; preeclamptic group (either mild or sever) and normal pregnancy; then compared them to healthy non-pregnant womens. The present study revealed that PT and APTT of PE group were significantly higher than normal pregnant group $(\mathrm{p}<0.05)$. Fibrinogen of the PE group was not significantly differed with normal pregnant group $(\mathrm{p} ; 0.3)$. However, hypofibrinogenemia was observed in $16.6 \%$ of PE cases. Jahromi et al. (2009) analyzed blood sample of 50 cases of severe PE and found that the mean values of APTT $(\mathrm{p}<0.005)$ and fibrin degradation product (FDP) $(\mathrm{p}<0.001)$ were higher in preeclamptic patients. However, they didn't find statistical differences in the mean values of plasma fibrinogen and PT between the two groups ( $p>0.05)$ [22]. Their results agreed with this study except for PT value; this could be of the small sample size analyzed in our study. Anyhow; the mean value of PT in their findings was higher in PE group than normal pregnant group. Moreover, their significance variation for this parameter between the two groups was borderline ( $\mathrm{p} ; 0.06)$.

Our results also revealed that $3(10 \%)$ of our severe PE cases had prolonged PT (mean: $16 \mathrm{sec})$ and APTT(mean: $42 \mathrm{sec}$ ) with hypofibrinogenemia(mean: $2.1 \mathrm{~g} / \mathrm{l}$ ). Jahromi et al. (2009) found that among their 25 PE patients, 3 cases showed evidence of disseminated intravascular coagulation (DIC) in their hospital course and had simultaneous prolongation of APTT and one patient had an elevated FDP [22].

Another study done by Han et al., (2014) who evaluated blood coagulation parameters and platelet indices during early and late pregnancy in 3 groups of subjects, normal pregnant women (n579), mild PE(n553) and severe PE (n542). They found that during late pregnancy in the normal pregnancy group, the APTT, PT, TT and platelet count decreased, while the fibrinogen level and mean platelet volume (MPV) increased compared to early pregnancy $(\mathrm{p}, 0.05)$. However, the PE patients presented with increased APTT, TT, MPV and D-dimer (DD) during late pregnancy [23].Their results matched with our findings in which the normal late pregnancy showed a physiological hypercoagulable state with an increased level of Fibrinogen . The explanation of this could be due to platelet consumption and aggregation followed by a secondary regeneration. However, the onset of $\mathrm{PE}$, in particular SPE, mainly results in complex disorders in the endogenous but not exogenous coagulative pathway, which results in the consumption of platelets and fibrinogen, with subsequent feedback activation of thrombopoiesis and fibrinolysis. Therefore, in the late pregnancy, PE patients present with a superhypercoagulable state along with the prolongation of APTT and TT, the augmentation of D-dimer (DD) and the enlargement of mean platelet volume (MPV) [23, 24].

However, these findings indicate that investigation of coagulation parameters are useful to detect an early ongoing coagulability in patients with severe PE who will develop DIC. However, more studies with large sample size should be conducted to screen and verify which coagulation parameters can predict the development of coagulability in such patients.

\section{V.Conclusion}

At late stage of pregnancy, the APTT, PT prolonged in PE pregnancy compared to normal pregnancy. However fibrinogen decreased in some cases of severe PE.

\section{Acknowledgements}

Authors would like to thank all subjects who were enrolled in this study both patients and controls. Special thanks to all medical staff in Omdurman Maternity Hospital for their help and cooperation.

\section{References}

[1]. Sibai B, Dekker G, Kupferminc M. Preeclampsia. Lancet, 365, 2005, 785-799.

[2]. [2] Stekkinger E, Zandstra M, Peeters LL, Spaandernen ME. Early-onset preeclampsia and the prevalence of postpartum metabolic syndrome. Obstet Gynaecol, 114(5), 2009, 1076-1084. 
[3]. Semenovakaya Z and Erogul M.. Pregnancy, Preeclampsia. eMedicine - Medical Reference, 2010, http://emedicine.medscape.com/article/796690-overview

[4]. Khan KS, Wojdyla D, Say L, Gülmezoglu AM, Van Look P. WHO analysis of causes of maternal death: a systemic review. Lancet, 367(9516), 2006, 1066-1074.

[5]. Duley L. The global impact of pre-eclampsia and eclampsia. Semin Perinatol, 33, 2009, 130-137.

[6]. [6] Osungbade KO and Ige OK. 'Public Health Perspectives of Preeclampsia in Developing Countries: Implication for Health System Strengthening. J of Preg, 2011, Article ID 481095.

[7]. Ali AA, Okud A, Khojali A, Adam I. High incidence of obstetric complications in Kassala hospital, eastern Sudan. J Obstet Gynaecol, 32(2), 2012, 148-149.

[8]. Roberts J, Gammil H. Preeclapsia: recent insight. Hypertension, 46(6), 2005, 1243-1249.

[9]. Fernando A, Daftary SN, Bhide AG. Hypertensive disorders in pregnancy. Practical guide to high risk pregnancy and delivery. $3^{\text {rd }}$ ed. New Delhi: Elsevier, 2008, p. 411.

[10]. Redman CW, Sargent IL. Placental stress and pre-eclampsia: a revised view. Placenta, 30, 2009, S38-42.

[11]. Fatemeh T, Marziyeh G, Nayereh G, Anahita G, Samira T. Maternal and perinatal outcome in nulliparious women complicated with pregnancy hypertension. J Pak Med Assoc, 60, 2010, 707-710.

[12]. Pennington KA, Schlitt JM, Jackson DL, Schulz LC, Schust DJ. Preeclampsia: multiple approaches for a multifactorial disease. Dis Model Mech, 5, 2012, 9-18.

[13]. Bremme KA. Haemostatic changes in pregnancy. Best Pract Res Clin Haematol, 16, 2003, $153-168$.

[14]. Dahlman T, Hellgren M \& Blomba ck M. Changes in blood coagulation and fibrinolysis in the normal puerperium. Gynecol Obstet Invest, 20, 1985, 37-44.

[15]. O'Riordan MN \& Higgins JR. Haemostasis in normal and abnormal pregnancy. Best Pract Res Clin Obstet Gynaecol, 17, 2003, 385-396.

[16]. Sattar N, Greer IA, Rumley A et al. A longitudinal study of the relationships between haemostatic, lipid and oestradiol changes during normal pregnancy. Thromb Haemost, 81, 1999, 71-75

[17]. Townsley DM. Hematologic complications of pregnancy. Semin Hematol, 50, 2013, 222-231.

[18]. Irminger-Finger I, Jastrow N, Irion O. Preeclampsia: a danger growing in disguise. Int J Biochem Cell Biol, 40, $2008,1979-1983$.

[19]. Metz J, Cincotta R, Francis M, De- Rosa L, Balloch A. Screening for consumptive coagulopathy in pree- clampsia. Int J Gynecol Obstet, 46, 1994, 3-9.

[20]. Barron WM, Heckerling P, Hibbard JU, Fisher S. Reducing unnecessary coagulation testing in hypertensive disorders of pregnancy. Obstet Gy necol, 94, 1999, 364-370.

[21]. Vladareanu AM, Andrei C, Onisai M, et al. The endothelial-platelet dysfunction in preeclampsia. Maedica- J of Clin Med, 2(3), 2007, 214-221.

[22]. B Namavar Jahromi , SH Rafiee. Coagulation Factors in Severe Preeclampsia . IRCMJ, 11(3), 2009, 321-324.

[23]. Han L, Liu X, Li H, Zou J, Yang Z, et al. Blood Coagulation Parameters and Platelet Indices: Changes in Normal and Preeclamptic Pregnancies and Predictive Values for Preeclampsia. PLoS ONE, 9(12), 2014, e114488.

[24]. Awad-Elkareem Abass, Remaz Abdalla, Isra Omer, Salma Ahmed, Ahoud Khalid, Husham Elzein. Evaluation of Platelets Count and Indices in Pre-Eclampsia Compared to Normal Pregnancies. IOSR J of Dent and Med Scien, 15(7), 2016, 05-08. 